\title{
The anxiety of not knowing: Diagnosis uncertainty about COVID-19
}

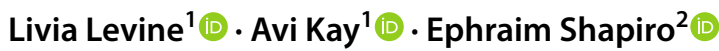

Accepted: 23 January 2022

(C) The Author(s), under exclusive licence to Springer Science+Business Media, LLC, part of Springer Nature 2022

\begin{abstract}
The emergence and swift global spread of COVID-19 brought increased anxiety worldwide (Santabárbara et al. (Progress in Neuro-Psychopharmacology \& Biological Psychiatry, 109, 110207, 2021)). Research regarding the COVID-19 outbreak addressed factors that contribute to anxiety people experienced as they tried to handle the changes in their lives associated with COVID-19 (Holmes et al. (The Lancet Psychiatry, 7(6), 547-560, 2020)). This paper focuses on diagnosis uncertainty as a particular source of anxiety. We use self-reported anxiety measures to understand how different stressors, and particularly how being sick or being unsure if one or one's close friends or relatives are sick, relate to overall anxiety levels. Fivehundred and thirty-three participants from a country with a stringent COVID-19 testing policy were surveyed in the spring of 2020 on various aspects of their anxiety and risk for depression, as well as on whether they or their friends or family had COVID-19. Analysis of survey results found that anxiety related to uncertainty regarding whether the survey responder or their friends or family were carrying COVID-19 may be even greater than fear of the virus itself. This paper discusses directional issues related to this finding and offers policy implications for decreasing anxiety during pandemics for certain types of communities. In addition to the main findings regarding diagnosis uncertainty and anxiety, this paper's results also indicate the importance of providing participants with an option for "not sure" in closed questions and imply the increased knowledge that can be gained by analyzing an unsure response independently of "yes" or "no".
\end{abstract}

Keywords Anxiety $\cdot$ COVID-19 $\cdot$ Diagnosis $\cdot$ Testing $\cdot$ Uncertainty

\section{Introduction}

\section{COVID-19 Anxiety}

In some ways, the anxieties related to COVID-19 are similar to anxieties surrounding other pandemics, such as $\mathrm{H} 1 \mathrm{~N} 1$, Ebola, SARS, and Zika (Chakraborty, 2020; Garfin et al., 2020; Limandri, 2020), but in many ways, COVID-19 is markedly different from those pandemics (Durodié, 2020; Gavi.org, 2020; Holmes et al., 2020). Drawing on research related to those pandemics, we see how fear of getting sick or fear of the spread of disease can affect psychological wellbeing. However, COVID-19 has involved significantly more uncertainty than other pandemics (Romiti \& Talerico, 2021).

Livia Levine

LiviaLevine@gmail.com

1 Department of Business Management, Jerusalem College of Technology, Jerusalem, Israel

2 Department of Health Systems Management, Ariel University, Ariel, Israel
There are two important features of COVID-19 that make it different from previous pandemics, more difficult to control on the physical level, and compound some of the psychological stress surrounding the pandemic:

COVID-19 is transmitted very quickly, requiring widespread restrictive government policies such as isolations and lockdowns (Gates, 2020; Wilder-Smith et al., 2020). The need to isolate people affects mental health in many ways, as has been described by researchers over the last year, who point to the trauma caused by physical distancing and isolation (Limandri, 2020; Santabárbara et al., 2021). Loneliness, parenting challenges, unemployment, travel restrictions, and general uncertainty all contribute to individuals' anxiety during this pandemic (Canady, 2020; Limandri, 2020; Rutter et al., 2020; Shapiro et al., 2020).

A second unique aspect of COVID-19 is that it is often unknown who is COVID-19 positive. In other recent global pandemics, there were very few asymptomatic carriers (Lee et al., 2003). In contrast, many people with COVID-19 are asymptomatic or have mild symptoms (Wilder-Smith et al., 2020). There even exists uncertainty about the uncertainty 
of the number of asymptomatic carriers; estimations of how many people with COVID-19 have mild or no symptoms range from 5-81 percent (Heneghan et al., 2020; Nogrady, 2020). Many of those with COVID-19-like symptoms often do not know whether to attribute them to COVID-19 or to a run-of-the-mill cough or cold. In addition to the anxiety related to being sick or the potential for being sick, COVID19 entails the additional stressor of not knowing if one is sick. This uncertainty emerged as a salient aspect of the survey we conducted.

\section{Uncertainty and Anxiety}

Anxiety from being "not sure" regarding COVID-19 diagnosis may stem more from general feelings of uncertainty rather than fear of consequences of the actual illness (Buheji et al., 2020; Clark, 2020). This may be particularly true among people with a high level of uncertainty avoidance, a measure that estimates the extent to which people try to avoid or feel threatened by ambiguous situations (Hofstede, 2020).

Studies from across the globe indicate a possible relationship between diagnosis uncertainty and anxiety. Survey data from Italy from the early months of COVID-19 (March 18-31, 2020) indicated that people who "do not know" whether they had been infected by COVID-19 or whether they had direct contact with infected people were more likely to have high anxiety symptoms (Forte et al., 2020). The above study was conducted during a time of high rates of infection in Italy, and $25.1 \%$ of their participants responded that they did not know if they have been infected. However, the authors do not isolate this data in their analysis.

Other researchers looked at aspects of uncertainty regarding COVID-19 diagnosis, but without isolating the specific issue of diagnosis uncertainty: Newby (Newby et al., 2020) found that $10.4 \%$ of Australians surveyed were unsure about their COVID-19 status, but they do not report whether that uncertainty affects anxiety. A study in the U.S. looked at uncertainty about susceptibility to COVID19 (Scarinci et al., 2021). A study of healthcare workers in Turkey showed that taking a COVID-19 test was related to increased anxiety and distress (Şahin et al., 2020). It is plausible that they took the test because of uncertainty about their COVID-19 status, but the authors do not specifically draw this conclusion. Researchers from China also suggest the idea that uncertainty about infection status may be a significant stressor for anxiety (Huang \& Zhao, 2020; Yu et al., 2020). However, in one of the above articles about China, the authors did not specifically measure this factor (Huang \& Zhao, 2020), and in the other, they combined all participants with "suspected" COVID-19, even if they were certain of their diagnosis (Yu et al., 2020). These and other studies published about COVID-19-related anxiety did not specifically address the mental health effects of uncertainty regarding COVID-19 diagnosis.

\section{Health Anxiety}

Recent research suggests that health anxiety is a significant factor in understanding COVID-19-related anxiety (Mertens et al., 2020; Nikčević et al., 2021; Sauer et al., 2020). People who are more anxious regarding their health to begin with are more likely to think that they are sick, not just that they might become sick. Taylor (Taylor, 2019) describes this phenomenon: "Highly publicized disease outbreaks can lead medically healthy individuals, especially those with high levels of health anxiety, to misinterpret benign bodily changes and sensations... as indicating that they have become infected, causing them to be unduly anxious" ( $\mathrm{p} 51$ ). Many are familiar with the effect of someone mentioning "head lice" and suddenly the regular tug of your hair feels like creatures are hiding in it. During pandemics, this effect means that someone who is generally anxious about health will read the description of COVID-19 symptoms into his body; this already anxious person is likely to feel a tingle in his throat and be concerned that he may have COVID-19.

\section{Testing and Uncertainty}

Laboratory tests can help quash COVID-19 diagnosis uncertainty. However, the tests are not available ubiquitously. This study, based on a survey of Israeli participants in the spring of 2020, examines a population with strict procedures surrounding testing. Israel has a national health system and a state-wide COVID-19 testing policy, which at the time of the survey only allowed people to get tested if they had a doctor's prescription. Israelis at that time did not have full access to COVID-19 tests, and therefore there were people who thought they may be COVID-19-positive who were not tested. In addition, Israel's policy of contacting and quarantining everyone who was in contact with a person with a positive COVID-19 test discouraged people from getting tested, even if they were unsure whether they had COVID19 , especially if they had no or mild symptoms. Israel's COVID-19 testing and quarantining policy at the time of the survey meant that there was a significant population who were unsure whether they had COVID-19 and who did not get tested.

\section{Method}

\section{Study Design}

Data was collected during the early part of the COVID-19 period, from April 22nd until May 11th, 2020, via an online 
questionnaire distributed to a convenience sample of adults in Israel. Israel was chosen as a test case because of its statewide COVID-19 testing policy and restricted availability. To increase sample size and representativeness, adult participants were recruited via a variety of community and religious groups, social media groups serving different cities, college students and alumni, and through a snowball effect of emails and social media posts to extended networks. A University Ethics Committee approved the survey.

The questionnaire was preceded by an informed consent form signed by all participants, which also included assurance of anonymity of survey responses, as well as a reminder to participants that the survey is not a substitute for professional medical advice.

The survey was offered in English and Hebrew translation, with the participants deciding on their preferred language. A professional translator ensured uniformity of the two versions.

The surveys were completed between April 22 - May 11, 2020, which was 6-8 weeks after the start of the COVID-19 lockdowns in Israel. By the end of the survey period, Israel reported 16,506 cases $(0.19 \%$ of the population) and 258 deaths due to COVID-19 (Worldometer - Real Time World Statistics, 2020).

\section{Measures}

The questionnaire included a series of questions related to anxiety and stress, answered on a Likert scale of 1 (none) to 5 (a lot). Questions included: Over the last two weeks, how much of a problem did you have with worry or anxiety?

Participants were also asked to complete previously validated scales, including the two question Patient Health Questionnaire (PHQ-2) depression screening tool (Mitchell et al., 2016), and an anxiety scale based on Wong et al., (Wong et al., 2007). The PHQ-2 is a widely accepted tool for understanding mental health. The Wong scale was specifically targeted to studying anxiety during pandemics. We adjusted the anxiety scale to adhere to cultural sensitivities regarding certain questions, and to limit survey exhaustion among participants. The full set of questions in the anxiety scale is available in the appendix.

We also asked participants to answer the questions "Have you or any of your friends or family been infected by corona virus?" The possible answers were: "yes," "no," or "not sure." Participants were also asked to rate their overall health and to answer questions about their specific worries related to COVID-19, based on related research related to epidemics (Wong et al., 2007).

Participants also provided demographic information, including country of birth, marital status, education, and whether they have children under the age of 10 or under the age of 18 .

\section{Statistical Analysis}

We analyzed the PHQ-2 and the anxiety scale as the sum of each of its components, weighted equally, with each question coded as interval variables. We used the anxiety scale as our primary measure of anxiety in the analyses. We also ran regressions with the same independent variables but using the single "worry and anxiety over the last two weeks" question as the dependent variable.

Survey results were analyzed using Python Jupyter with Statsmodels (Seabold \& Perktold, 2010). Median comparisons and ordinal logistic regressions were used to understand relationships between variables. For the regression analyses, we chose control variables that we hypothesized would impact anxiety levels. We then removed variables that contributed neither to the model fit, nor to the theoretical understanding of the survey results.

The primary analysis uses the results from all the participants who answered the relevant questions. Prior research indicates a correlation between health anxiety and major depression symptoms (Scarella et al., 2016). In order to understand anxiety among participants who were less likely to have health anxiety, we conducted a secondary analysis of the participants who were not at high risk for depression, defined as a score of less than three on the two-question Patient Health Questionnaire (PHQ-2) aggregate (Mitchell et al., 2016). We used the same control variables in the low depression-risk analysis as we did in the analysis involving all the participants.

\section{Results}

Five-hundred-and-thirty-three participants answered the survey.

\section{Demographics}

Sixty-two percent of the sample was female and $50 \%$ born in Israel. The average age of the participants was 48 , with $74 \%$ of the participants married, $50 \%$ with children under 18 , and $11 \%$ with children under 10 . Sixty-nine percent of the participants have a bachelor's degree.

\section{Anxiety}

The median self-reported "worry and anxiety" measure over the last two weeks before the survey was 2 (on a scale of $1-5$, , with $24 \%$ of participants reporting high or very high worry and anxiety ( 4 or 5 ). The median anxiety scale aggregate was 7 (on a scale of 4-16). Twenty percent of participants scored 10 or above on the aggregate anxiety scale. Cronbach's alpha for the 4-question anxiety scale was 0.73 . 
Table 1 General anxiety and specific corona-related stressors in Israel

\begin{tabular}{|c|c|c|c|c|c|c|c|c|}
\hline & \multicolumn{2}{|l|}{ Overall } & \multicolumn{2}{|c|}{$\begin{array}{l}\text { "Not sure" if I or } \\
\text { friends or family are } \\
\text { sick }\end{array}$} & \multicolumn{2}{|c|}{$\begin{array}{l}\text { I or friends or family } \\
\text { are sick }\end{array}$} & \multicolumn{2}{|c|}{$\begin{array}{l}\text { I am not sick, nor are } \\
\text { friends or family }\end{array}$} \\
\hline & Median & $\%$ of 4 or 5 & Median & $\%$ of 4 or 5 & Mean & $\%$ of 4 or 5 & Median & $\%$ of 4 or 5 \\
\hline Worry or anxiety in past 2 weeks & 2.00 & 0.24 & 4.00 & 0.53 & 2.00 & 0.22 & 2.00 & 0.22 \\
\hline Afraid to go to hospital & 3.00 & 0.41 & 4.00 & 0.53 & 3.00 & 0.46 & 3.00 & 0.38 \\
\hline Worry that family and friends will be infected & 3.00 & 0.46 & 4.00 & 0.60 & 4.00 & 0.54 & 3.00 & 0.42 \\
\hline Afraid daily life will be interfered with & 3.00 & 0.30 & 4.00 & 0.53 & 3.00 & 0.29 & 3.00 & 0.29 \\
\hline $\begin{array}{l}\text { Feel that government failing to provide } \\
\text { adequate information }\end{array}$ & 3.00 & 0.33 & 3.00 & 0.40 & 2.00 & 0.26 & 3.00 & 0.35 \\
\hline Concern for own health or health of family & 3.00 & 0.42 & 4.00 & 0.70 & 4.00 & 0.54 & 3.00 & 0.35 \\
\hline
\end{tabular}

The specific stressors which were reported as the most worrisome were concerns about personal health and the health of friends and family. This concern manifested itself in both specific worries about family and friends being infected by COVID-19 (median 3 on a scale of 1-5) as well as generalized concern for health (median 3 on a scale of 1-5). The list of stressors addressed in the survey is reported in Table 1.

\section{Demographic Factors and Anxiety}

Certain demographic variables had significant relationships with anxiety levels, as measured by the aggregate anxiety scale. Women reported significantly higher anxiety than men $(p<.001)$ and anxiety was lower in older participants $(p<.001)$.

We did not find any association between education and anxiety, nor did including education in the regression model significantly change the impact of the other variables.

People with children under age 10 at home reported higher anxiety compared to people with older children (aged 10-18) and compared to people without children at home $(p<.05)$ Table 2 and Table 3.

Table 2 Logistic ordinal regression on aggregate anxiety scale

\begin{tabular}{lll}
\hline & Coef & $95 \%$ confidence interval \\
\hline Not sure & 0.50 & $-0.20-1.20$ \\
No corona & $0.42^{*}$ & $0.06-0.78$ \\
Female & $0.68^{* * *}$ & $0.36-1.00$ \\
Child under 10 & $0.55^{*}$ & $0.02-1.07$ \\
Age & $-0.02^{* * *}$ & $-0.03--0.74$ \\
Constant & $1.02 * * *$ & $0.69-1.35$
\end{tabular}

$\mathrm{N}: 533 * p<0.05, * * p<0.01, * * * p<0.001$

\section{Diagnosis Uncertainty and Anxiety}

Participants answered the question "Have you or any of your friends or family been infected by corona virus" with "yes," "no," or "not sure." The respondents who answered "yes" did not show higher levels of anxiety than others. However, $30 \%$ of the participants who answered "not sure" scored 10 or above on the anxiety scale aggregate, compared to $19 \%$ for the rest of the sample. This effect was even stronger when we looked at the results to the single question about worry and anxiety over the last two weeks. Fifty-three percent of participants who were not sure if they or their family and friends have COVID-19 reported high or very high (4 or 5) worry and anxiety, compared to $22 \%$ for others (two-tailed ttest statistic $=3.16, p=0.002$ ).

Ordinal logistic regressions performed on the same data show that even while controlling for demographic variables mentioned above, being uncertain about COVID-19 diagnosis is related to higher anxiety levels, even though having COVID-19 is associated with lower anxiety levels. This effect is not significant, but the direction of the effect on the aggregate anxiety scale is still relevant. The relationship between uncertainty of COVID-19 diagnosis and the answer to the question "Over the last two weeks, how much of a problem did you have with worry or anxiety?" is significant

Table 3 Logistic ordinal regression on worry and anxiety over the two weeks prior to the survey

\begin{tabular}{lll}
\hline & Coef & $95 \%$ confidence interval \\
\hline Not sure & $0.81^{*}$ & $0.04-1.58$ \\
No corona & 0.20 & $-0.16-0.55$ \\
Female & $0.67 * * *$ & $0.35-1.00$ \\
Child under 10 & -0.12 & $-0.65-0.41$ \\
Age & $-0.01 * *$ & $-0.02--0.00$ \\
Constant & $1.36^{* * *}$ & $1.01-1.70$
\end{tabular}

$\mathrm{N}: 533 * p<0.05, * * p<0.01, * * * p<0.001$ 
$(p<.05)$ when using the same controlling variables (gender, age, and children under the age of 10).

\section{Health Anxiety}

After finding a relationship between anxiety and COVID19 status diagnosis uncertainty, we conducted a further analysis to better understand the direction of causality. We did not specifically ask about health anxiety, but we did ask questions related to depression risk, which has been shown in prior research to correlate with health anxiety (Scarella et al., 2016). Therefore, we tried to control for health anxiety by looking only at those who have a low risk for depression, and to see whether the relationship between anxiety and uncertainty still exists. To focus our study on the participants with average or strong general mental health, we analyzed the data in a similar way as we did above, but only looking at the 448 participants who were not at high risk for depression, defined as a score of less than three on the two-question Patient Health Questionnaire (PHQ-2) aggregate (Mitchell et al., 2016). In this subgroup, the 18 participants who were "not sure" about their COVID-19 diagnosis reported higher anxiety (median of 7 on the anxiety scale) than those who did not report diagnosis uncertainty (median of 6 on the anxiety scale). However, the association between "not sure" and the anxiety scale was not statistically significant in regressions, possibly due to the small number of uncertain respondents in this category (Table 4).

\section{Discussion}

This study examined the relationship between uncertainty regarding COVID-19 diagnosis and anxiety. One of the surprising results of this study was that people who were "not sure" whether they or their family or friends had been infected were more likely to have high anxiety than people (or friends or family) who were actually sick, and people who were sick (or friends or family who were sick) did not have notably high anxiety. We offer two possible explanations for this. The first is that people have anxiety about

Table 4 Logistic ordinal regression on aggregate anxiety scale for participants with low PHQ-2 (low risk of depression)

\begin{tabular}{lll}
\hline & Coef & $95 \%$ confidence interval \\
\hline Not sure & 0.45 & $-0.39-1.28$ \\
No corona & $0.40^{*}$ & $0.01-0.79$ \\
Female & $0.59^{* *}$ & $0.25-0.94$ \\
Child under 10 & $0.71 *$ & $0.13-1.29$ \\
Age & $-0.02 * *$ & $-0.03--0.01$ \\
Constant & $0.87 * * *$ & $0.51-1.23$ \\
\hline
\end{tabular}

$\mathrm{N}: 447 * p<0.05, * * p<0.01, * * * p<0.001$ possibly being sick and this is even scarier than actually being sick. Not knowing whether you have COVID-19 or not also means you do not know how or whether it will progress, whether you will need treatment, and whether you will be able to receive satisfactory treatment. All these unknowns generate anxiety.

Another explanation is that people with higher health anxiety are more likely to report general anxiety and are also more likely to be unsure about their COVID-19 diagnosis. The direction of the results in the secondary analysis indicates that health anxiety does not necessarily explain the original results. However the lack of statistical significance in this subgroup lends support to the explanation that predisposition to anxiety generates the feeling of diagnosis uncertainty.

While our findings support the idea that uncertainty about COVID-19 diagnosis is related to anxiety, we still do not know if the uncertainty is a catalyst for high anxiety or if it feeds the anxiety, and we suggest this as an area for further research.

Although they may neither exhibit physical signs of having COVID-19, and the risk of their becoming physically sick or getting others physically sick may be minimal, the people who are uncertain whether they have been infected present a mental health risk and should be tested in order to address their mental health needs.

The implications of this work extend beyond the current COVID-19 crisis, as there are many times when people are not sure whether they have an illness (Gheihman et al., 2020). Often the decision whether to test for an illness is based on a cost-benefit analysis that compares the cost of testing to the potential benefit of a correct diagnosis for treating the physical illness (Nakase-Richardson et al., 2020; Rowthorn \& Maciejowski, 2020; Sun et al., 2019). Based on the findings of this paper, providers and healthcare decision makers should consider the mental health effects of diagnosis uncertainty in the cost-benefit analyses. Testing can be used to help decrease anxiety among people who are uncertain whether they are sick, whether the illness is a global pandemic or any other illness.

\section{Anxiety as a Social Feature}

The demographic findings in the survey were in-line with previous research. Israelis place a high value on family and community and therefore are likely to experience high anxiety with issues surrounding their family and community members' health (Schwartz et al., 2000; Shapiro et al., 2021). Previous research on anxiety in Israel found that even before COVID-19, women and younger people reported higher anxiety than men and older people, respectively (Averbuch, 2021). Also, it is not surprising that parents of young children had higher anxiety following a long 
lockdown during which schools and daycare centers were closed.

\section{Uncertainty as a Social Feature}

People from different communities have differing levels of propensity for uncertainty in their lives (Schwartz \& Melech, 2000). The Israelis surveyed in this study are accustomed to uncertainty as a part of their life in general (de Bruijn et al., 2020) and tend to have high levels of worry about themselves and the people around them (Schwartz \& Melech, 2000). Many parents during the COVID-19 lockdowns may have recalled their own experiences as children being required to stay inside for weeks during the Gulf War thirty years prior. Additionally, various security threats mean many Israelis are familiar with uncertainty. And research shows that Israelis have a relatively low tolerance for uncertainty (Hofstede, 2020). It is possible that uncertainty is more of a trigger for Israelis than it is for others, specifically because of its consistency in Israelis' daily lives.

Uncertainty is a regular part of life for people in many communities, in addition to the Israeli ones studied here. There are countries whose health systems were not adequately serving their communities even before COVID-19 hit, and for whom uncertainty regarding health-related issues is a constant (Noor \& Islam, 2020). There are communities that lack food and water, (Nephawe et al., 2021) or other basic needs, and therefore live in constant uncertainty. There are socio-cultural factors in many areas that may affect the relationship between diagnosis uncertainty and anxiety, such as social identification and trust (Paolini et al., 2020). And there are factors that could increase uncertainty about health issues within a population, such as gender, race, history, and education (Scarinci et al., 2021; Shuwiekh et al., 2020). Each of these groups is prone to constant uncertainty, similar to the group surveyed in this paper. These populations are possibly especially prone to exhibit a relationship between diagnosis uncertainty and anxiety like the one we saw in Israel.

\section{Limitations \& Future Research}

There are several limitations to this work. The survey may have some degree of selection bias. The survey was conducted using a snowball method and is not necessarily fully representative of the Israeli public. The surveys were also limited to those who were comfortable answering in either English or Hebrew.

All the survey data used in this paper was self-reported and may not accurately represent objective anxiety levels. Included in the difficulties of self-reported data are biases related to Likert scales, such as central tendency bias and acquiescence bias. To mitigate these distortions, we used validated questions and aggregate response scales, including questions that were reverse-coded. To mitigate the social desirability bias we confirmed the anonymity of responses in both the request to take the survey and in the survey itself. An additional difficulty with data collection is that the crosssectional survey only asked about anxiety at one point in time, so we do not know how anxiety levels of participants compare to their anxiety levels before COVID-19.

Future research should investigate the direction of the uncertainty-anxiety connection, such as through longitudinal research. In addition, while these surveys all took place during the first wave of COVID-19, future research can look at the long-term viability of the uncertainty-anxiety effect. Furthermore, including additional countries in this research may afford a more exact understanding of the matter at hand.

\section{Conclusion}

This research suggests that not being sure whether one has been infected by COVID-19 can be significantly linked to higher anxiety. From our analyses we saw that uncertainty was more strongly related to greater anxiety than even having the disease. This implies that increasing testing capabilities, and particularly encouraging people who think they might be infected to get tested, can have a positive impact on the individuals' mental health.

In the short term, as the number of variants increases, the uncertainty regarding their symptoms among vaccinated and unvaccinated individuals rises, which in turn increases diagnosis uncertainty. In the long term, this finding regarding the relationship between uncertainty and anxiety can be applied to other medical concerns and suggests that a focus on testing effectiveness and availability is critical for public mental health.

An important lesson to be learned from this analysis is about how researchers frame survey questions. As noted, there have been many studies about COVID-19-related anxiety over the last year, and in nearly all of them participants were asked whether they or their friends and family have contracted the disease. However, respondents typically only had the options to respond with "yes" or "no." By also including a "Not sure" option, researchers can allow participants to report their situations more accurately. Analyzing this additional information can allow for a more complete and nuanced understanding of the impact of uncertainty on the mental health of individuals.

\section{Appendix: Selected Survey Questions}

Single-question Worry-Anxiety: Over the last 2 weeks, how much of a problem did you have with worry or anxiety? Answered on a scale of 1-5 
Patient Health Questionnaire-2 (Mitchell et al., 2016,) which asked participants to indicate whether they were bothered by the following problems over the two weeks before the survey on a scale of 1-4 (not at all, several days, more than half the days, nearly every day):

Little interest or pleasure in doing things

Feeling down, depressed or hopeless

Anxiety scale based on Wong, Gao and Tam, 2006, in which we asked participants "To what extent do the following apply to you" on a scale of 1-4 (never/very rarely, sometimes, often, very often/always):

I feel nervous and anxious

I get upset easily or feel panicky

I feel like I'm falling apart and going to pieces

I feel calm (reverse-coded)

Fears about the virus itself: "To what degree do the following apply to you" on a scale of 1-5:

I am afraid to go to the hospital for consultation.

I worry that my family members and friends will be infected

I feel the government fails to provide adequate information about the corona virus

The restrictions on my daily life have caused me stress To what degree are you concerned about either your personal health or the health of your friends and family due to the corona virus outbreak?

To what degree are you concerned about your personal economic situation due to the corona virus outbreak?

Authors' Contributions All authors contributed significantly to this paper. The three authors contributed to writing the survey and collecting the data. All authors participated in the theory development of the paper, led by the first author. The first author was responsible for the analysis of the raw data and writing of the paper. All authors assisted in the literature review and in editing the paper.

Funding The research was self-funded by the authors.

Availability of Data and Material (Data Transparency) The data that support the findings of this study are available from the corresponding author upon request.

Code Availability (Software Application or Custom Code) All Python analysis codes used in the paper are available from the corresponding author upon request.

\section{Declarations}

Ethics Approval (Include Appropriate Approvals or Waivers) The Ethics Committee from Ariel University approved the survey. Reference \# AU-HEA-ES-20200412
Consent to Participate (Include Appropriate Consent Statements) All participants clicked the button associated with the words: "By clicking on the button, I provide my informed consent to participate in this survey and to permit use of the anonymous data I provide. I understand that I can discontinue my participation in this survey at any time."

Consent for Publication (Consent Statement Regarding Publishing an Individual's Data or Image) Not relevant

Conflicts of Interest/Competing Interests None

\section{References}

Averbuch, E. (2021). Facts and approaches to health inequality in Israel 2020. Israel Ministy of Health.

Buheji, M., Ahmed, D., \& Jahrami, H. (2020). Living uncertainty in the new normal. International Journal of Applied Psychology, $10(2), 21-31$

Canady, V. A. (2020). APA stress report amid COVID-19 points to parental challenges. Mental Health Weekly, 30(22), 3-4. https:// doi.org/10.1002/mhw. 32385

Chakraborty, N. (2020). The COVID-19 pandemic and its impact on mental health. Progress in Neurology and Psychiatry, 24(2), 21-24. https://doi.org/10.1002/pnp.666

Clark, D. (2020). Living with uncertainty during COVID-19. Psychology Today. https://www.psychologytoday.com/us/blog/the-runaw ay-mind/202003/living-uncertainty-during-covid-19. Accessed 2020

de Bruijn, A. L., Feldman, Y., Kuiper, M. E., Brownlee, M., Reinders Folmer, C., Kooistra, E. B., Olthuis, E., Fine, A., \& van Rooij, B. (2020). Why did Israelis comply with COVID-19 Mitigation Measures during the initial first wave lockdown? Available at SSRN 3681964

Durodié, B. (2020). Handling uncertainty and ambiguity in the COVID-19 pandemic. Psychological Trauma: Theory, Research, Practice, and Policy, 12(S1), S61.

Forte, G., Favieri, F., Tambelli, R., \& Casagrande, M. (2020). The enemy which sealed the world: Effects of COVID-19 diffusion on the psychological state of the Italian population. Journal of Clinical Medicine, 9(6), 1802. https://doi.org/10.3390/jcm9061802

Garfin, D. R., Silver, R. C., \& Holman, E. A. (2020). The novel coronavirus (COVID-2019) outbreak: Amplification of public health consequences by media exposure. Health Psychology. 39(5) 355

Gates, B. (2020). Responding to Covid-19-A Once-in-a-Century Pandemic? I NEJM. New England Journal of Medicine. https://doi. org/10.1056/nejmp2003762

Gavi.org. (2020). How does COVID-19 compare to past pandemics?. https://www.gavi.org/vaccineswork/how-does-covid-19compare-past-pandemics. Accessed 2020

Gheihman, G., Johnson, M., \& Simpkin, A. L. (2020). Twelve tips for thriving in the face of clinical uncertainty. Medical Teacher, 42(5), 493-499.

Heneghan, C., Brassey, J., \& Jefferson, T. (2020). COVID-19: What proportion are asymptomatic? CEBM. https://www.cebm.net/ covid-19/covid-19-what-proportion-are-asymptomatic/. Accessed 2020

Hofstede. (2020). Country Comparison Tool. Hofstede Insights. https:// www.hofstede-insights.com/. Accessed 2020

Holmes, E. A., O'Connor, R. C., Perry, V. H., Tracey, I., Wessely, S., Arseneault, L., Ballard, C., Christensen, H., Silver, R. C., Everall, I., Ford, T., John, A., Kabir, T., King, K., Madan, I., Michie, S., Przybylski, A. K., Shafran, R., Sweeney, A., ... Bullmore, E. (2020). Multidisciplinary research priorities for the COVID-19 
pandemic: A call for action for mental health science. The Lancet Psychiatry, 7(6), 547-560https://doi.org/10.1016/S2215-0366(20) 30168-1

Huang, Y., \& Zhao, N. (2020). Generalized anxiety disorder, depressive symptoms and sleep quality during COVID-19 outbreak in China: A web-based cross-sectional survey. Psychiatry Research, 288,112954

Lee, H. K., Tso, E. Y., Chau, T. N., \& Tsang, O. T. (2003). Asymptomatic severe acute respiratory syndrome-associated coronavirus infection. Emerging Infectious Diseases, 9(11), 1491.

Limandri, B. J. (2020). Pandemic anxiety: How can I help? Journal of Psychosocial Nursing and Mental Health Services, 58(7), 7-10.

Mertens, G., Gerritsen, L., Duijndam, S., Salemink, E., \& Engelhard, I. M. (2020). Fear of the coronavirus (COVID-19): Predictors in an online study conducted in March 2020. Journal of Anxiety Disorders, 74, 102258.

Mitchell, A. J., Yadegarfar, M., Gill, J., \& Stubbs, B. (2016). Case finding and screening clinical utility of the Patient Health Questionnaire (PHQ-9 and PHQ-2) for depression in primary care: A diagnostic meta-analysis of 40 studies. BJPsych Open, 2(2), 127-138.

Nakase-Richardson, R., Hoffman, J. M., Magalang, U., Almeida, E., Schwartz, D. J., Drasher-Phillips, L., Ketchum, J. M., Whyte, J., Bogner, J., \& Dismuke-Greer, C. E. (2020). Cost-benefit analysis from the payor's perspective for the screening and diagnosing OSA during inpatient rehabilitation for moderate to severe TBI. Archives of Physical Medicine and Rehabilitation.

Nephawe, N., Mwale, M., Zuwarimwe, J., \& Tjale, M. M. (2021). The impact of water-related challenges on rural communities food security initiatives. AGRARIS: Journal of Agribusiness and Rural Development Research, 7(1), 11-23.

Newby, J., O’Moore, K., Tang, S., Christensen, H., \& Faasse, K. (2020). Acute mental health responses during the COVID-19 pandemic in Australia. PloS one, 15(7), e0236562.

Nikčević, A. V., Marino, C., Kolubinski, D. C., Leach, D., \& Spada, M. M. (2021). Modelling the contribution of the Big Five personality traits, health anxiety, and COVID-19 psychological distress to generalised anxiety and depressive symptoms during the COVID-19 pandemic. Journal of Affective Disorders, 279, 578-584. https://doi.org/10.1016/j.jad.2020.10.053

Nogrady, B. (2020). What the data say about asymptomatic COVID infections. Nature, 587(7835), 534-535. https://doi.org/10.1038/ d41586-020-03141-3

Noor, F. M., \& Islam, Md. M. (2020). Prevalence and associated risk factors of mortality among COVID-19 patients: A meta-analysis. Journal of Community Health, 45(6), 1270-1282. https://doi.org/ 10.1007/s10900-020-00920-x

Paolini, D., Maricchiolo, F., Pacilli, M. G., \& Pagliaro, S. (2020). COVID-19 lockdown in Italy: The role of social identification and social and political trust on well-being and distress. Current Psychology, 1-8.

Romiti, G. F., \& Talerico, G. (2021). Embracing the uncertainty: An important lesson from COVID-19. Journal of General Internal Medicine. https://doi.org/10.1007/s11606-021-06809-2

Rowthorn, R., \& Maciejowski, J. (2020). A cost-benefit analysis of the COVID-19 disease. Oxford Review of Economic Policy, 36(Supplement_1), S38-S55.

Rutter, H., Wolpert, M., \& Greenhalgh, T. (2020). Managing uncertainty in the covid-19 era. Bmj, 370 .

Şahin, M. K., Aker, S., Şahin, G., \& Karabekiroğlu, A. (2020). Prevalence of depression, anxiety, distress and insomnia and related factors in healthcare workers during COVID-19 pandemic in Turkey. Journal of Community Health, 45(6), 1168-1177.

Santabárbara, J., Lasheras, I., Lipnicki, D. M., Bueno-Notivol, J., Pérez-Moreno, M., López-Antón, R., De la Cámara, C., Lobo, A.,
\& Gracia-García, P. (2021). Prevalence of anxiety in the COVID19 pandemic: An updated meta-analysis of community-based studies. Progress in Neuro-Psychopharmacology \& Biological Psychiatry, 109, 110207. https://doi.org/10.1016/j.pnpbp.2020. 110207

Sauer, K. S., Jungmann, S. M., \& Witthöft, M. (2020). Emotional and behavioral consequences of the COVID-19 pandemic: The role of health anxiety, intolerance of uncertainty, and distress (in) tolerance. International Journal of Environmental Research and Public Health, 17(19), 7241.

Scarella, T. M., Laferton, J. A. C., Ahern, D. K., Fallon, B. A., \& Barsky, A. (2016). The relationship of hypochondriasis to anxiety, depressive, and somatoform disorders. Psychosomatics, 57(2), 200-207. https://doi.org/10.1016/j.psym.2015.10.006

Scarinci, I. C., Pandya, V. N., Kim, Y., Bae, S., Peral, S., Tipre, M., Hardy, C., Hansen, B., \& Baskin, M. L. (2021). Factors associated with perceived susceptibility to COVID-19 among urban and rural adults in Alabama. Journal of Community Health. https://doi.org/ 10.1007/s10900-021-00976-3

Schwartz, S. H., \& Melech, G. (2000). National differences in micro and macro worry: Social, economic, and cultural explanations. In Culture and subjective well-being (p. 40). The MIT Press.

Schwartz, S. H., Sagiv, L., \& Boehnke, K. (2000). Worries and values. Journal of Personality, 68(2), 309-346. https://doi.org/10.1111/ 1467-6494.00099

Seabold, S., \& Perktold, J. (2010). Statsmodels: Econometric and statistical modeling with python. Proceedings of the 9th Python in Science Conference, 57, 61.

Shapiro, E., Levine, L., \& Kay, A. (2020). Mental health stressors in Israel during the coronavirus pandemic. Psychological Trauma: Theory, Research, Practice, and Policy, 12(5), 499. https://doi. org/10.1037/tra0000864

Shapiro, E., Levine, L., \& Kay, A. (2021). A Jerusalem synagogue coping during the coronavirus pandemic: Challenges and Opportunities. Journal of Religion and Health, 60(1), 81-98.

Shuwiekh, H. A., Kira, I. A., Sous, M. S. F., Ashby, J. S., Alhuwailah, A., Baali, S. B. A., Azdaou, C., Oliemat, E. M., \& Jamil, H. J. (2020). The differential mental health impact of COVID-19 in Arab countries. Current Psychology, 1-15.

Sun, L., Brentnall, A., Patel, S., Buist, D. S., Bowles, E. J., Evans, D. G. R., Eccles, D., Hopper, J., Li, S., \& Southey, M. (2019). A cost-effectiveness analysis of multigene testing for all patients with breast cancer. JAMA Oncology, 5(12), 1718-1730.

Taylor, S. (2019). The psychology of pandemics: Preparing for the next global outbreak of infectious disease. Cambridge Scholars Publishing.

Wilder-Smith, A., Chiew, C. J., \& Lee, V. J. (2020). Can we contain the COVID-19 outbreak with the same measures as for SARS? The Lancet Infectious Diseases.

Wong, T. W., Gao, Y., \& Tam, W. W. S. (2007). Anxiety among university students during the SARS epidemic in Hong Kong. Stress and Health: Journal of the International Society for the Investigation of Stress, 23(1), 31-35.

Worldometer-Real time world statistics. (2020). https://www.world ometers.info/. Accessed 2020

Yu, H., Li, M., Li, Z., Xiang, W., Yuan, Y., Liu, Y., Li, Z., \& Xiong, Z. (2020). Coping style, social support and psychological distress in the general Chinese population in the early stages of the COVID19 epidemic. BMC Psychiatry, 20, 426. https://doi.org/10.1186/ s12888-020-02826-3

Publisher's note Springer Nature remains neutral with regard to jurisdictional claims in published maps and institutional affiliations. 